\title{
Sliding Mode Leader Following Control for Autonomous Air Robots
}

\author{
Touraj Soleymani, Student Member, IEEE, and Fariborz Saghafi
}

\begin{abstract}
In this paper, we propose a leader following control for autonomous air robots. The separated design strategy with kinematic acceleration commands is used. The location of the robot with respect to the leader is specified by a range and two angles. We obtain the kinematic model of the system represented by the state-space equations. The controller is designed based on the sliding mode control which asymptotically stabilizes the tracking errors in presence of uncertainties and disturbances. In order to implement the leader following controller in the air robots, a control system is introduced which converts the acceleration commands to the actuator commands. Simulations are provided to show the performance of the leader following control.
\end{abstract}

Index Terms-Air refueling, autonomous air robots, formation flight, leader following, sliding mode control.

\section{INTRODUCTION}

$\mathrm{T}$ HE objective of the leader following control is following a leader and maintaining a specific relative position with respect to it. In the aeronautical application, a follower can be considered as an autonomous air robot and the leader can be any flying object its motion information is measured by the follower. In the leader following control, the control of the follower is of our interest. Applications of leader following control include formation flight [1], [2], [3], air refueling [4], etc.

The leader following problem for a robot can be viewed as the motion control problem. The challenge in the motion control problem is how to control the robot in a manner that its kinematic states track the desired ones. Generally, the motion control studies on the autonomous air vehicles are based on two design strategies. One is the integrated design [5] in which the motion controller generates directly the actuators commands. The other is separated design [6] in which it is assumed that the vehicle is equipped with an inner control system, traditionally called autopilot; the motion controller generates steering commands and the vehicle's control system generates the actuators commands in order to track them.

Different commands can be used in the separated strategy and correspondingly the vehicle is required to equip with the appropriate control system. One type is the velocity or acceleration kinematic commends which can be either

Touraj Soleymani is with Department of Aerospace Engineering, Sharif University of Technology, Tehran, Iran (e-mail: touraj@ieee.org).

Fariborz Saghafi is with Department of Aerospace Engineering, Sharif University of Technology, Tehran, Iran (e-mail: saghafi@sharif.edu) expressed in Cartesian or the spherical coordinate systems. The common examples of this type are the combinations of speed, heading angle, and flight path angle commands [7] or latax commands in the guidance laws [8]. Other types are simplified performance commands like angle of attack, bank angle, and thrust commands [9].

Here, the leader following controller is designed for autonomous air robots based on the separated strategy with acceleration kinematic commands in the spherical coordinate system. This provides the follower to approach the leader in a desired direction and avoid collision with the leader. The approach direction is considered along the leader's velocity vector. The position of the follower with respect to the leader is specified by a range and two angles called azimuth and elevation.

We develop a framework for the leader following control represented by the state-space equations of the kinematic system. We introduce two essential frames called line of approach and line of sight and taking into account the rotations of frames with respect to the inertial reference frame in the development of the time derivatives.

In addition, we employ the sliding mode control [10] to design the leader following controller. The sliding mode control is a robust nonlinear control that can attenuate the effects of the uncertainty and disturbances. The equivalent control and saturation function are employed in order to eliminate the chattering phenomenon. The application of the sliding mode control for ground robots appears in [11], [12].

The rest of the paper is as follows. In Section II the kinematic model is developed. Section III proposes the leader following control based on the sliding mode control. Section VI describes the vehicle's control system. The simulations and results are provided in Section V. Finally, a conclusion is given is Section VI.

\section{KINEMATIC MODELING}

In this section, we develop the appropriate equations which will be used in the leader following control design. We employ Fig. 1 to illustrate the geometry of the leader following through this paper where $B, T$, and $E$ denote respectively the follower's center of mass, the leader's center of mass, and the inertial reference point. The inertial reference frame $E$ is generated by the point $E$ and the triad $\boldsymbol{e}_{1}, \boldsymbol{e}_{2}, \boldsymbol{e}_{3}$ and the local level coordinate system $L$ is associated with the inertial reference frame. The follower frame $B$ is generated by the point $B$ and the triad $\boldsymbol{b}_{1}, \boldsymbol{b}_{2}, \boldsymbol{b}_{3}$ 
and the body coordinate system $B$ is associated with the follower frame. Since the objective is to develop a controller for the follower, we call it the vehicle for the simplicity. In this study, vectors and tensors are respectively written in the bold lowercase and uppercase letters; with superscripts and subscripts representing the associated frames and points respectively. We use brackets with a superscript to express the quantity coordinates of a vector or a tensor in a specific coordinate system.

It is assumed that the vehicle can measure the location of the leader $\left[\boldsymbol{s}_{T B}\right]^{B}$, the velocity of the leader $\left[\boldsymbol{v}_{T}^{B}\right]^{B}$, and the acceleration of the leader $\left[\boldsymbol{a}_{T}^{B}\right]^{B}$; all with respect to itself and in the body coordinate system via sensors. It measures its location with respect to the inertial reference point $\left[\boldsymbol{s}_{B E}\right]^{L}$ and its velocity with respect to the inertial reference frame $\left[\boldsymbol{v}_{B}^{E}\right]^{L}$; both in the local level coordinate system. The transformation matrix between the body and the local level coordinate systems $[\boldsymbol{T}]^{B L}$, the angular velocity of the vehicle with respect to the inertial frame in the body coordinate system $\left[\boldsymbol{\omega}^{B E}\right]^{B}$, and the rate of it $\left[\dot{\boldsymbol{\omega}}^{B E}\right]^{B}$ are also measured. However, some may be indirectly obtained by numerical computations.

First, we acquire our appropriate ingredients based on the available information. The relative location of the vehicle with respect to the leader in the local level coordinate system is given by

$$
\left[\boldsymbol{s}_{B T}\right]^{L}=-[\boldsymbol{T}]^{L B}\left[\boldsymbol{s}_{T B}\right]^{B}
$$

The inertial differential velocity of the vehicle with respects to the leader is obtained as

$$
\boldsymbol{v}_{B T}^{E}=D^{E} \boldsymbol{s}_{B T}=-D^{E} \boldsymbol{s}_{T B}=-D^{B} \boldsymbol{s}_{T B}-\boldsymbol{\Omega}^{B E} \boldsymbol{s}_{T B}
$$

where $D^{X}$ is the rotational time derivative operator which maintains the tensor properties even with respect to time varying frames (for more information see [13]) and $\boldsymbol{\Omega}^{B E}$ is the skew symmetric matrix of the angular velocity $\boldsymbol{\omega}^{B E}$. Expressing (2) in the local level coordinate system, we have

$$
\left[\boldsymbol{v}_{B T}^{E}\right]^{L}=-[\boldsymbol{T}]^{L B}\left(\left[\boldsymbol{v}_{T}^{B}\right]^{B}+\left[\boldsymbol{\Omega}^{B E}\right]^{B}[\boldsymbol{T}]^{B L}\left[\boldsymbol{s}_{T B}\right]^{B}\right)
$$

The inertial differential acceleration of the vehicle with respect to the leader is obtained as

$$
\begin{aligned}
& \boldsymbol{a}_{B T}^{E}=D^{E} D^{E} \boldsymbol{s}_{B T}=-D^{E} D^{E} \boldsymbol{s}_{T B}=-D^{E}\left(D^{B} \boldsymbol{s}_{T B}+\boldsymbol{\Omega}^{B E} \boldsymbol{s}_{T B}\right) \\
& =-D^{B} D^{B} \boldsymbol{s}_{T B}-2 \boldsymbol{\Omega}^{B E} D^{B} \boldsymbol{s}_{T B}-D^{B} \boldsymbol{\Omega}^{B E} \boldsymbol{s}_{T B}-\boldsymbol{\Omega}^{B E} \boldsymbol{\Omega}^{B E} \boldsymbol{s}_{T B}
\end{aligned}
$$

Expressing (4) in the local level coordinate system, we have

$$
\begin{aligned}
& {\left[\boldsymbol{a}_{B T}^{E}\right]^{L}=-[\boldsymbol{T}]^{L B}\left(\left[\boldsymbol{a}_{T}^{B}\right]^{B}+2\left[\boldsymbol{\Omega}^{B E}\right]^{B}[\boldsymbol{T}]^{B L}\left[\boldsymbol{v}_{T}^{B}\right]^{B}\right.} \\
& \left.+\left[\dot{\boldsymbol{\Omega}}^{B E}\right]^{B}[\boldsymbol{T}]^{L B}\left[\boldsymbol{s}_{T B}\right]^{B}+\left[\boldsymbol{\Omega}^{B E}\right]^{B}\left[\boldsymbol{\Omega}^{B E}\right]^{B}[\boldsymbol{T}]^{B L}\left[\boldsymbol{s}_{T B}\right]^{B}\right)
\end{aligned}
$$

The velocity of the leader with respect to the inertial frame is obtained as

$$
\boldsymbol{v}_{T}^{E}=\boldsymbol{v}_{B}^{E}-\boldsymbol{v}_{B T}^{E}
$$

and its coordinates in the local level coordinate system is

$$
\left[\boldsymbol{v}_{T}^{E}\right]^{L}=\left[\begin{array}{lll}
v_{x} & v_{y} & v_{z}
\end{array}\right]^{T}
$$

We define the line of approach frame and its associated coordinate system (see Fig.1) in a way the coordinates of $\boldsymbol{v}_{T}^{E}$ in the line of approach coordinate system are written as

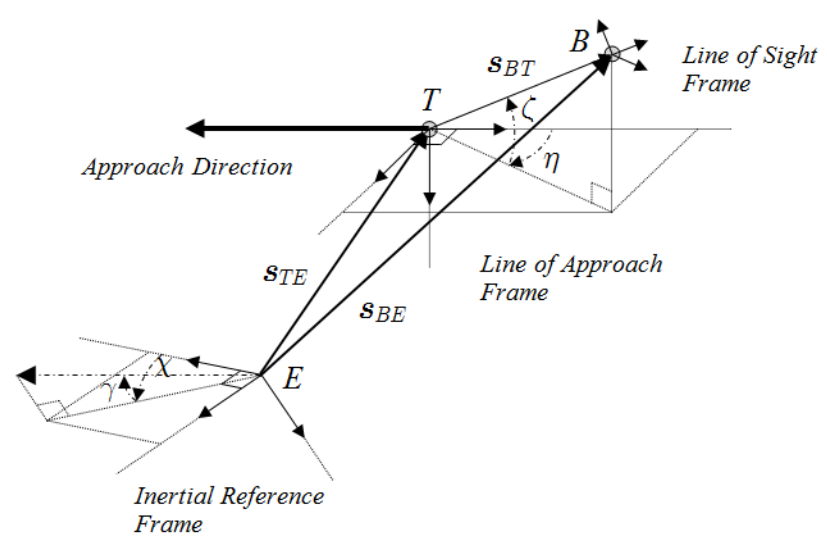

Fig. 1. Geometry of leader following problem.

$$
\left[\boldsymbol{v}_{T}^{E}\right]^{A}=\left[\begin{array}{lll}
-V & 0 & 0
\end{array}\right]^{T}
$$

where

$$
V=\sqrt{v_{x}{ }^{2}+v_{y}{ }^{2}+v_{z}{ }^{2}}
$$

The transformation matrix between the line of approach and the local level coordinate systems is

$$
[\boldsymbol{T}]^{A L}=[\boldsymbol{T}]^{A Y}[\boldsymbol{T}]^{Y X}[\boldsymbol{T}]^{X L}=\left[\begin{array}{ccc}
-\mathrm{c} \chi \mathrm{c} \gamma & -\mathrm{s} \chi \mathrm{c} \gamma & \mathrm{s} \gamma \\
\mathrm{s} \chi & -\mathrm{c} \chi & 0 \\
\mathrm{c} \chi \mathrm{s} \gamma & \mathrm{s} \chi \mathrm{s} \gamma & \mathrm{c} \gamma
\end{array}\right]
$$

where $X$ and $Y$ are intermediate coordinate system, $c($. stands for cosine, $s($.$) stands for sine and$

$$
\begin{gathered}
{[\boldsymbol{T}]^{X L}=\left[\begin{array}{ccc}
\mathrm{c} \chi & \mathrm{s} \chi & 0 \\
-\mathrm{s} \chi & \mathrm{c} \chi & 0 \\
0 & 0 & 1
\end{array}\right], \quad[\boldsymbol{T}]^{Y X}=\left[\begin{array}{ccc}
\mathrm{c} \gamma & 0 & -\mathrm{s} \gamma \\
0 & 1 & 0 \\
\mathrm{~s} \gamma & 0 & \mathrm{c} \gamma
\end{array}\right]} \\
{[\boldsymbol{T}]^{X L}=\left[\begin{array}{ccc}
-1 & 0 & 0 \\
0 & -1 & 0 \\
0 & 0 & 1
\end{array}\right]}
\end{gathered}
$$

and $\chi$ denotes the heading angle and $\gamma$ denotes the flight path angle that are defined as

$$
\chi=\operatorname{atan} 2\left(v_{y}, v_{x}\right), \quad \gamma=\operatorname{atan}\left(\frac{-v_{z}}{\sqrt{v_{x}{ }^{2}+v_{y}{ }^{2}}}\right)
$$

The location of the vehicle with respect to the leader is

$$
\left[\boldsymbol{s}_{B T}\right]^{A}=[\boldsymbol{T}]^{A L}\left[\boldsymbol{s}_{B T}\right]^{L}
$$

and its coordinates in the line of approach coordinate system can be denoted as

$$
\left[\boldsymbol{s}_{B T}\right]^{A}=\left[\begin{array}{lll}
x & y & z
\end{array}\right]^{T}
$$

The line of sight frame and its associated coordinate system (see Fig.1) are defined in a manner that the coordinates of $s_{B T}$ in the line of sight coordinate system are written as

$$
\left[\boldsymbol{s}_{B T}\right]^{C}=\left[\begin{array}{lll}
\rho & 0 & 0
\end{array}\right]^{T}
$$

where

$$
\rho=\sqrt{x^{2}+y^{2}+z^{2}}
$$

The transformation matrix between the line of sight and the line of approach coordinate systems is 


$$
[\boldsymbol{T}]^{C A}=[\boldsymbol{T}]^{C Z}[\boldsymbol{T}]^{Z A}=\left[\begin{array}{ccc}
\mathrm{c} \eta \mathrm{c} \zeta & \mathrm{s} \eta \mathrm{c} \zeta & -\mathrm{s} \zeta \\
-\mathrm{s} \eta & \mathrm{c} \eta & 0 \\
\mathrm{c} \eta \mathrm{s} \zeta & \mathrm{s} \eta \mathrm{s} \zeta & \mathrm{c} \zeta
\end{array}\right]
$$

where $Z$ is an intermediate coordinate system and

$$
[\boldsymbol{T}]^{Z A}=\left[\begin{array}{ccc}
\mathrm{c} \eta & \mathrm{s} \eta & 0 \\
-\mathrm{s} \eta & \mathrm{c} \eta & 0 \\
0 & 0 & 1
\end{array}\right], \quad[\boldsymbol{T}]^{C Z}=\left[\begin{array}{ccc}
\mathrm{c} \zeta & 0 & -\mathrm{s} \zeta \\
0 & 1 & 0 \\
\mathrm{~s} \zeta & 0 & \mathrm{c} \zeta
\end{array}\right]
$$

and $\eta$ denotes the azimuth angle and $\zeta$ denotes the elevation angle which are defined as

$$
\eta=\operatorname{atan} 2(y, x), \quad \zeta=\operatorname{atan}\left(\frac{-z}{\sqrt{x^{2}+y^{2}}}\right)
$$

The angular velocity of line of sight frame with respect to the inertial frame can be written as

$$
\boldsymbol{\omega}^{C E}=\boldsymbol{\omega}^{C A}+\boldsymbol{\omega}^{A L}
$$

where

$$
\begin{aligned}
\boldsymbol{\omega}^{C A} & =\dot{\zeta} \boldsymbol{y}_{C}+\dot{\eta} \boldsymbol{z}_{Z} \\
\boldsymbol{\omega}^{A L} & =\dot{\gamma} \boldsymbol{y}_{Y}+\dot{\chi} \boldsymbol{z}_{X}
\end{aligned}
$$

Expressing (21) in the appropriate coordinate systems, we have

$$
\begin{gathered}
{\left[\boldsymbol{\omega}^{C A}\right]^{C}=\dot{\zeta}\left[\begin{array}{l}
0 \\
1 \\
0
\end{array}\right]+\dot{\eta}[\boldsymbol{T}]^{C Z}\left[\begin{array}{l}
0 \\
0 \\
1
\end{array}\right]} \\
{\left[\boldsymbol{\omega}^{A L}\right]^{A}=\dot{\gamma}[\boldsymbol{T}]^{A L}\left[\begin{array}{l}
0 \\
1 \\
0
\end{array}\right]+\dot{\chi}[\boldsymbol{T}]^{A X}\left[\begin{array}{l}
0 \\
0 \\
1
\end{array}\right]}
\end{gathered}
$$

where $[\boldsymbol{T}]^{A X}=[\boldsymbol{T}]^{A Y}[\boldsymbol{T}]^{Y X}$. Thus, we can express (20) in the line of sight coordinate system

$$
\left[\boldsymbol{\omega}^{C E}\right]^{C}=\left[\boldsymbol{\omega}^{C A}\right]^{C}+[\boldsymbol{T}]^{C A}\left[\boldsymbol{\omega}^{A L}\right]^{A}
$$

Suppose that the coordinates of $\omega^{C E}$ in the line of sight coordinate system are

$$
\left[\boldsymbol{\omega}^{C E}\right]^{C}=\left[\begin{array}{lll}
\tilde{p} & \tilde{q} & \tilde{r}
\end{array}\right]
$$

By writing $\left[\boldsymbol{\omega}^{C E}\right]^{C}$ in the component form, we have

$$
\begin{aligned}
& \tilde{p}=-\dot{\eta} \mathrm{s} \zeta+\dot{\chi} \mathrm{s} \gamma \mathrm{c} \zeta \mathrm{c} \eta-\dot{\gamma} \mathrm{c} \zeta \mathrm{s} \eta-\dot{\chi} \mathrm{c} \gamma \mathrm{s} \zeta \\
& \tilde{q}=\dot{\zeta}-\dot{\chi} \mathrm{s} \gamma \mathrm{s} \eta-\dot{\gamma} \mathrm{c} \eta \\
& \tilde{r}=\dot{\eta} \mathrm{c} \zeta+\dot{\chi} \mathrm{s} \gamma \mathrm{s} \zeta \mathrm{c} \eta-\dot{\gamma} \mathrm{s} \zeta \mathrm{s} \eta+\dot{\chi} \mathrm{c} \gamma \mathrm{c} \zeta
\end{aligned}
$$

The acceleration of the leader with respect to the inertial frame is obtained as

$$
\boldsymbol{a}_{T}^{E}=\boldsymbol{a}_{B}^{E}-\boldsymbol{a}_{B T}^{E}
$$

and its coordinates in the local level coordinate system is written as

$$
\left[\boldsymbol{a}_{T}^{E}\right]^{L}=\left[\begin{array}{lll}
a_{x} & a_{y} & a_{z}
\end{array}\right]^{T}
$$

One can also obtain the inertial acceleration of the leader with respect to the line of sight frame as following

$$
\boldsymbol{a}_{T}^{E}=D^{E} \boldsymbol{v}_{T}^{E}=D^{A} \boldsymbol{v}_{T}^{E}+\boldsymbol{\Omega}^{A E} \boldsymbol{v}_{T}^{E}
$$

and express it in the line of approach coordinate system as

$$
\left[\boldsymbol{a}_{T}^{E}\right]^{A}=\left[\dot{\boldsymbol{v}}_{T}^{E}\right]^{A}+\left[\boldsymbol{\Omega}^{A E}\right]^{A}\left[\boldsymbol{v}_{T}^{E}\right]^{A}=\left[\begin{array}{c}
-\dot{V} \\
V \dot{\chi} \mathrm{c} \gamma \\
V \dot{\gamma}
\end{array}\right]
$$

Since we have the inertial acceleration of the leader in the local level coordinate system, we can write

$$
\left[\boldsymbol{a}_{T}^{E}\right]^{A}=[\boldsymbol{T}]^{A L}\left[\boldsymbol{a}_{T}^{E}\right]^{L}=\left[\begin{array}{ccc}
-\mathrm{c} \chi \mathrm{c} \gamma & -\mathrm{s} \chi \mathrm{c} \gamma & \mathrm{s} \gamma \\
\mathrm{s} \chi & -\mathrm{c} \chi & 0 \\
\mathrm{c} \chi \mathrm{s} \gamma & \mathrm{s} \chi \mathrm{s} \gamma & \mathrm{c} \gamma
\end{array}\right]\left[\begin{array}{l}
a_{x} \\
a_{y} \\
a_{z}
\end{array}\right]
$$

Thus, $\dot{\chi}$ and $\dot{\gamma}$ are obtained by equalizing (29) and (30)

$$
\begin{aligned}
& \dot{\chi}=\frac{a_{x} \mathrm{~s} \chi-a_{y} \mathrm{c} \chi}{V} \\
& \dot{\gamma}=\frac{a_{x} \mathrm{~s} \gamma \mathrm{c} \chi+a_{y} \mathrm{~s} \gamma \mathrm{s} \chi+a_{z} \mathrm{c} \gamma}{V}
\end{aligned}
$$

To obtain our kinematic equations, we express the inertial differential velocity and acceleration in the line of sight coordinate system. However, their derivatives should first be shifted from the inertial frame to the line of sight frame. For the inertial differential velocity, we have

$$
\boldsymbol{v}_{B T}^{E}=D^{E} \boldsymbol{s}_{B T}=D^{C} \boldsymbol{s}_{B T}+\boldsymbol{\Omega}^{C E} \boldsymbol{s}_{B T}
$$

One can write (33) in the line of sight coordinate system

$$
\left[\boldsymbol{v}_{B T}^{E}\right]^{C}=\left[\dot{\boldsymbol{s}}_{B T}\right]^{C}+\left[\boldsymbol{\Omega}^{C E}\right]^{C}\left[\boldsymbol{s}_{B T}\right]^{C}=\left[\begin{array}{c}
\dot{\rho} \\
\rho \tilde{r} \\
-\rho \tilde{q}
\end{array}\right]
$$

For the inertial differential acceleration, we have

$$
\begin{aligned}
& \boldsymbol{a}_{B T}^{E}=D^{E} D^{E} \boldsymbol{s}_{B T}=D^{E}\left(D^{C} \boldsymbol{s}_{B T}+\boldsymbol{\Omega}^{C E} D^{E} \boldsymbol{s}_{B T}\right) \\
& =D^{C} D^{C} \boldsymbol{s}_{B T}+2 \boldsymbol{\Omega}^{C E} D^{C} \boldsymbol{s}_{B T}+D^{C} \boldsymbol{\Omega}^{C E} \boldsymbol{s}_{B T}+\boldsymbol{\Omega}^{C E} \boldsymbol{\Omega}^{C E} \boldsymbol{s}_{B T}
\end{aligned}
$$

By expressing (34) in the line of sight coordinate system, we have

$$
\begin{aligned}
& {\left[\boldsymbol{a}_{B T}^{E}\right]^{C}=\left[\ddot{\boldsymbol{s}}_{B T}\right]^{C}+2\left[\boldsymbol{\Omega}^{C E}\right]^{C}\left[\dot{\boldsymbol{s}}_{B T}\right]^{C}+\left[\dot{\boldsymbol{\Omega}}^{C E}\right]^{C}\left[\boldsymbol{s}_{B T}\right]^{C}} \\
& +\left[\boldsymbol{\Omega}^{C E}\right]^{C}\left[\boldsymbol{\Omega}^{C E}\right]^{C}\left[\boldsymbol{s}_{B T}\right]^{C}=\left[\begin{array}{c}
\ddot{\rho}-\rho \tilde{r}^{2}-\rho \tilde{q}^{2} \\
\rho \dot{\tilde{r}}+2 \dot{\rho} \tilde{r}+\rho \tilde{p} \tilde{q} \\
-\rho \dot{\tilde{q}}-2 \dot{\rho} \tilde{q}+\rho \tilde{p} \tilde{r}
\end{array}\right]
\end{aligned}
$$

\section{LEADER Following CONTROL}

The objective of the leader following control is to maintain the relative distance of the vehicle $\rho$ at a desired distance $d$ and the azimuth angle $\eta$ at desired azimuth $\eta$ and the elevation angle $\zeta$ at the desired elevation $\zeta_{e}$ in a manner that the vehicle approaches the leader in a desired direction specified by the leader's velocity. We employ the sliding mode control to design the leader following controller which is to generate acceleration commands in the line of sight coordinate system to steer the vehicle. In the sliding mode control, we first require to obtain the system's dynamic equations. The kinematic equations for the inertial differential velocity and acceleration developed in the preceding section are used in obtaining these equations.

The coordinates of the inertial differential velocity in the line of sight coordinate system are 


$$
\left[\boldsymbol{v}_{B}^{E}\right]^{C}=\left[\begin{array}{lll}
V_{\rho} & V_{\eta} & V_{\zeta}
\end{array}\right]^{T}
$$

It follows from (33) that

$$
\begin{aligned}
& V_{\rho}=\dot{\rho} \\
& V_{\eta}=\rho \tilde{r} \\
& V_{\zeta}=-\rho \tilde{q}
\end{aligned}
$$

One can decompose $\tilde{p}, \tilde{q}$, and $\tilde{r}$ in (25) as following

$$
\begin{aligned}
& \tilde{p}=-\dot{\eta} \mathrm{s} \zeta+\hat{p} \\
& \tilde{q}=\dot{\zeta}+\hat{q} \\
& \tilde{r}=\dot{\eta} \mathrm{c} \zeta+\hat{r}
\end{aligned}
$$

Thus, by substituting (38) in (37) and solving for $\dot{\rho}, \dot{\eta}$, and $\dot{\zeta}$, we have

$$
\left\{\begin{array}{l}
\dot{\rho}=V_{\rho} \\
\dot{\eta}=\frac{V_{\eta}-\rho \hat{r}}{\rho \cos (\zeta)} \\
\dot{\zeta}=-\frac{V_{\zeta}+\rho \hat{q}}{\rho}
\end{array}\right.
$$

The vehicle acceleration is considered as the input and the leader acceleration is generally uncertain and can be viewed as bounded disturbances. The coordinates of the inertial acceleration of the vehicle and leader in the line of sight coordinate system are

$$
\left[\boldsymbol{a}_{B}^{E}\right]^{C}=\left[\begin{array}{lll}
u_{1} & u_{2} & u_{3}
\end{array}\right]^{T}, \quad\left[\begin{array}{l}
\boldsymbol{a}_{T}^{E}
\end{array}\right]^{C}=\left[\begin{array}{lll}
\delta_{1} & \delta_{2} & \delta_{3}
\end{array}\right]^{T}
$$

It follows from (35) that

$$
\begin{aligned}
& u_{1}-\delta_{1}=\ddot{\rho}-\rho \tilde{r}^{2}-\rho \tilde{q}^{2} \\
& u_{2}-\delta_{2}=\rho \dot{\tilde{r}}+2 \dot{\rho} \tilde{r}+\rho \tilde{p} \tilde{q} \\
& u_{3}-\delta_{3}=-\rho \dot{\tilde{q}}-2 \dot{\rho} \tilde{q}+\rho \tilde{p} \tilde{r}
\end{aligned}
$$

Following from the derivative of (37), we have

$$
\begin{aligned}
& \ddot{\rho}=\dot{V}_{\rho} \\
& \dot{\tilde{r}}=\frac{\dot{V}_{\eta}-\dot{\rho} \tilde{r}}{\rho} \\
& \dot{\tilde{q}}=-\frac{\dot{V_{\zeta}}-\dot{\rho} \tilde{q}}{\rho}
\end{aligned}
$$

and using (39) one can rewrites (38)

$$
\begin{aligned}
& \tilde{p}=-\frac{V_{\eta}-\rho \hat{r}}{\rho \cos (\zeta)} \mathrm{s} \zeta+\hat{p} \\
& \tilde{q}=-\frac{V_{\zeta}+\rho \hat{q}}{\rho}+\hat{q} \\
& \tilde{r}=\frac{V_{\eta}-\rho \hat{r}}{\rho \cos (\zeta)} \mathrm{c} \zeta+\hat{r}
\end{aligned}
$$

By substituting (42) and (43) in (41) and solving for $\dot{V}_{\rho}, \dot{V}_{\eta}$, and $\dot{V}_{\zeta}$, we have

$$
\left\{\begin{array}{l}
\dot{V}_{\rho}=\frac{V_{\eta}^{2}}{\rho}+\frac{V_{\zeta}^{2}}{\rho}-\delta_{1}+u_{1} \\
\dot{V}_{\eta}=-\frac{V_{\rho} V_{\eta}}{\rho}+\left(\frac{-V_{\eta}+\rho \hat{r}}{\rho} \tan \zeta+\hat{p}\right) V_{\zeta}-\delta_{2}+u_{2} \\
\dot{V}_{\zeta}=-\frac{V_{\rho} V_{\zeta}}{\rho}-\left(\frac{-V_{\eta}+\rho \hat{r}}{\rho} \tan \zeta+\hat{p}\right) V_{\eta}-\delta_{3}+u_{3}
\end{array}\right.
$$

To shift the open-loop equilibrium point in absence of the uncertainty to origin, we use the following change of variables

$$
\begin{array}{lll}
\rho_{e}=\rho-d, & \dot{\rho}_{e}=\dot{\rho}, & \ddot{\rho}_{e}=\ddot{\rho} \\
\eta_{e}=\eta-\eta_{d}, & \dot{\eta}_{e}=\dot{\eta}, & \ddot{\eta}_{e}=\ddot{\eta} \\
\zeta_{e}=\zeta-\zeta_{d}, & \dot{\zeta}_{e}=\dot{\zeta}, & \ddot{\zeta}_{e}=\ddot{\zeta}
\end{array}
$$

Clearly, one can write (39) and (44) in the following general state space form

$$
\left\{\begin{array}{l}
\dot{\boldsymbol{x}}=\boldsymbol{f}_{a}(\boldsymbol{x}, \boldsymbol{y}, \boldsymbol{w}) \\
\dot{\boldsymbol{y}}=\boldsymbol{f}_{b}(\boldsymbol{x}, \boldsymbol{y}, \boldsymbol{w})-\boldsymbol{\delta}(\boldsymbol{x}, \boldsymbol{w})+\boldsymbol{u}
\end{array}\right.
$$

where

$$
\begin{gathered}
\boldsymbol{x}=\left[\begin{array}{l}
\rho_{e} \\
\eta_{e} \\
\zeta_{e}
\end{array}\right], \quad \boldsymbol{y}=\left[\begin{array}{l}
V_{\rho} \\
V_{\eta} \\
V_{\zeta}
\end{array}\right], \quad \boldsymbol{w}=\left[\begin{array}{l}
\chi \\
\gamma \\
\dot{\chi} \\
\dot{\gamma}
\end{array}\right], \quad \boldsymbol{u}=\left[\begin{array}{l}
u_{1} \\
u_{2} \\
u_{3}
\end{array}\right], \quad \boldsymbol{\delta}=\left[\begin{array}{l}
\delta_{1} \\
\delta_{2} \\
\delta_{3}
\end{array}\right] \\
\boldsymbol{f}_{a}=\left[\begin{array}{c}
V_{\rho} \\
\frac{V_{\eta}-\left(\rho_{e}+d\right) \hat{r}}{\left(\rho_{e}+d\right) \cos \left(\zeta+\zeta_{e}\right)} \\
\frac{V_{\zeta}+\left(\rho_{e}+d\right) \hat{q}}{-\left(\rho_{e}+d\right)}
\end{array}\right] \\
\boldsymbol{f}_{b}=\left[\begin{array}{c}
\frac{V_{\eta}{ }^{2}}{\left(\rho_{e}+d\right)}+\frac{V_{\zeta}{ }^{2}}{\left(\rho_{e}+d\right)} \\
-\frac{V_{\rho} V_{\eta}}{\left(\rho_{e}+d\right)}+\left(\frac{-V_{\eta}+\left(\rho_{e}+d\right) \hat{r}}{\left(\rho_{e}+d\right)} \tan \left(\zeta+\zeta_{e}\right)+\hat{p}\right) V_{\zeta} \\
-\frac{V_{\rho} V_{\zeta}}{\left(\rho_{e}+d\right)}-\left(\frac{-V_{\eta}+\left(\rho_{e}+d\right) \hat{r}}{\left(\rho_{e}+d\right)} \tan \left(\zeta+\zeta_{e}\right)+\hat{p}\right) V_{\eta}
\end{array}\right]
\end{gathered}
$$

where $\boldsymbol{w}$ includes the leader's velocity and acceleration information and $\hat{p}, \hat{q}$, and $\hat{r}$ are all functions of $\boldsymbol{x}$ and $\boldsymbol{w}$ and if they are unknown they are regarded as bounded disturbances. Our goal is to design a state feedback control to stabilize the origin for all uncertainties. Note that (46) is in the regular form so the sliding mode control can be applied. We design the sliding manifolds as following

$$
s=\boldsymbol{y}-\phi(x)
$$

where

$$
\boldsymbol{\phi}(\boldsymbol{x})=-\left[\begin{array}{ccc}
k_{1} & 0 & 0 \\
0 & k_{2} & 0 \\
0 & 0 & k_{3}
\end{array}\right] \boldsymbol{x}
$$

and $k_{1}, k_{2}$, and $k_{3}$ are constants which stabilize the following reduced-order system

$$
\dot{x}=f_{a}(x, \phi(x))
$$

Differentiation of (48) and substitution of (46) yields

$$
\dot{\boldsymbol{s}}=\dot{\boldsymbol{y}}-\frac{\partial \boldsymbol{\phi}(\boldsymbol{x})}{\partial \boldsymbol{x}} \dot{\boldsymbol{x}}=\boldsymbol{f}_{b}(\boldsymbol{x}, \boldsymbol{y}, \boldsymbol{w})-\boldsymbol{\delta}(\boldsymbol{x}, \boldsymbol{w})+\boldsymbol{u}-\frac{\partial \phi(\boldsymbol{x})}{\partial \boldsymbol{x}} \boldsymbol{y}
$$

Hence, the equivalent control is obtained as

$$
\boldsymbol{u}_{e}=-\boldsymbol{f}_{b}(\boldsymbol{x}, \boldsymbol{y}, \boldsymbol{w})+\boldsymbol{\delta}(\boldsymbol{x}, \boldsymbol{w})+\frac{\partial \phi(\boldsymbol{y})}{\partial \boldsymbol{y}} \boldsymbol{x}
$$

which results in $\dot{s}=\mathbf{0}$. The uncertainty control is considered as 


$$
\boldsymbol{u}_{s}=-\boldsymbol{B} \operatorname{sat}\left(\boldsymbol{E}^{-1} \boldsymbol{s}\right)
$$

where

$$
\boldsymbol{B}=\left[\begin{array}{ccc}
\beta_{1} & 0 & 0 \\
0 & \beta_{2} & 0 \\
0 & 0 & \beta_{3}
\end{array}\right], \quad \boldsymbol{E}=\left[\begin{array}{ccc}
\varepsilon_{1} & 0 & 0 \\
0 & \varepsilon_{2} & 0 \\
0 & 0 & \varepsilon_{3}
\end{array}\right], \quad \operatorname{sat}(\boldsymbol{z})=\left[\begin{array}{c}
\operatorname{sat}\left(z_{1}\right) \\
\operatorname{sat}\left(z_{2}\right) \\
\operatorname{sat}\left(z_{3}\right)
\end{array}\right]
$$

and $\beta_{i}$ and $\varepsilon_{i}$ are positive constants and $\operatorname{sat}(z)$ denotes the saturation function. Note that $\beta_{i}$ is selected in a manner that the reaching condition is satisfied. Thus, the sliding mode control can be expressed as

$$
\boldsymbol{u}=-\boldsymbol{f}_{b}(\boldsymbol{x}, \boldsymbol{y}, \boldsymbol{w})+\boldsymbol{\delta}(\boldsymbol{x}, \boldsymbol{w})+\frac{\partial \phi(\boldsymbol{y})}{\partial \boldsymbol{y}} \boldsymbol{x}-\boldsymbol{B} \operatorname{sat}\left(\boldsymbol{E}^{-1} \boldsymbol{s}\right)
$$

Therefore, the acceleration command can be written in terms of components of the sliding mode control as following

$$
\left[\hat{\boldsymbol{a}}_{c}\right]^{C}=\left[\begin{array}{lll}
u_{1} & u_{2} & u_{3}
\end{array}\right]^{T}
$$

which is the command used by the control system.

\section{VEHICLE'S CONTROL SYSTEM}

The objective of the vehicle's control system is to track the generated acceleration command and stabilize the vehicle's dynamics. Since the command is expressed in the line of sight coordinate system, it must first be written in the local level coordinate system

$$
\left[\hat{\boldsymbol{a}}_{c}\right]^{L}=[\boldsymbol{T}]^{L C}\left[\hat{\boldsymbol{a}}_{c}\right]^{C}
$$

where $[\boldsymbol{T}]^{L C}$ is the transformation matrix between the line of sight and the local level coordinate systems given by

$$
[\boldsymbol{T}]^{C L}=[\boldsymbol{T}]^{C A}[\boldsymbol{T}]^{A L}
$$

To take into account the effect of gravity, the command must be compensated by the gravitational acceleration

$$
\left[\boldsymbol{a}_{c}\right]^{L}=\left[\hat{\boldsymbol{a}}_{c}\right]^{L}-[\boldsymbol{g}]^{L}
$$

where $[g]^{L}$ is the gravitational acceleration that works as a bias. To make the acceleration command usable for the vehicle, it must be transformed to the body coordinate system

$$
\left[\boldsymbol{a}_{c}\right]^{B}=[\boldsymbol{T}]^{B L}\left[\boldsymbol{a}_{c}\right]^{L}
$$

where $[\boldsymbol{T}]^{B L}$ is the transformation matrix between the local level and the body coordinate systems written in terms of the Euler's angles

$$
[\boldsymbol{T}]^{L B}=\left[\begin{array}{ccc}
\mathrm{c} \psi \mathrm{c} \theta & \mathrm{s} \psi \mathrm{c} \theta & -\mathrm{s} \theta \\
\mathrm{c} \psi \mathrm{s} \theta \mathrm{s} \phi-\mathrm{s} \psi \mathrm{c} \phi & \mathrm{s} \psi \mathrm{s} \theta \mathrm{s} \phi+\mathrm{c} \psi \mathrm{c} \phi & \mathrm{c} \theta \mathrm{s} \phi \\
\mathrm{c} \psi \mathrm{s} \theta \mathrm{c} \phi+\mathrm{s} \psi \mathrm{s} \phi & \mathrm{s} \psi \mathrm{s} \theta \mathrm{c} \phi-\mathrm{c} \psi \mathrm{s} \phi & \mathrm{c} \theta \mathrm{c} \phi
\end{array}\right]
$$

The coordinates of the acceleration command in the body coordinate system can be expressed as

$$
\left[\boldsymbol{a}_{c}\right]^{B}=\left[\begin{array}{lll}
\tilde{a}_{x c} & \tilde{a}_{y c} & \tilde{a}_{z c}
\end{array}\right]^{T}
$$

where $\tilde{a}_{x c}, \tilde{a}_{y c}$, and $\tilde{a}_{z c}$ denote the axial, lateral, and normal acceleration commands respectively. The acceleration commands associated with the bank to turn vehicles is obtained as

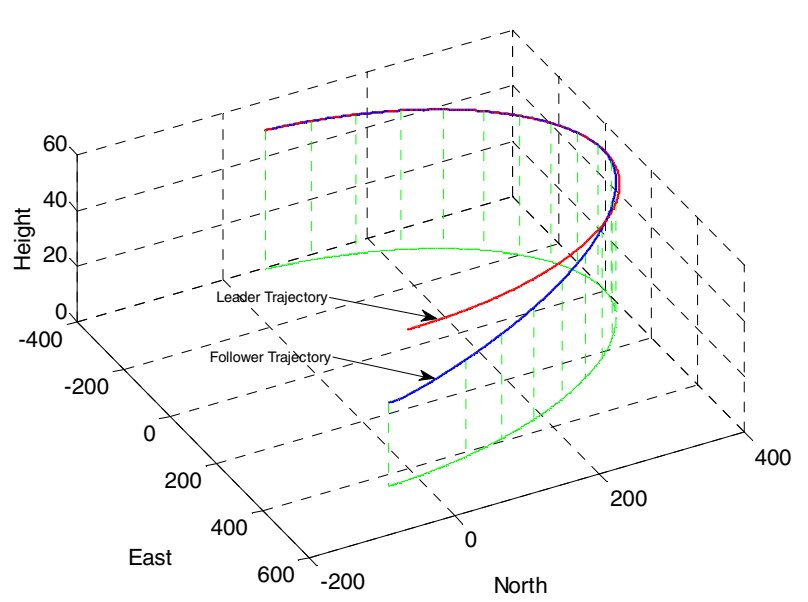

Fig. 2. Trajectories of leader and follower.

$$
\begin{gathered}
a_{x c}=\tilde{a}_{x c}, \quad a_{z c}=\operatorname{sgn}\left(\tilde{a}_{z c}\right) \sqrt{\left(\tilde{a}_{y c}\right)^{2}+\left(\tilde{a}_{z c}\right)^{2}} \\
, \quad \phi_{c}=\hat{\phi}_{c}+\phi
\end{gathered}
$$

where $\operatorname{sgn}(z)$ is the signum function, $\phi$ is the current roll angle of the vehicle, and $\hat{\phi}_{c}$ is the roll error angle command defined as

$$
\hat{\phi}_{c}=-\tan ^{-1}\left(\frac{a_{y c}^{S}}{a_{z c}^{S}}\right)
$$

Clearly, $\left|\hat{\phi}_{c}\right| \leq 90^{\circ}$. Thus, for the maneuvers, the vehicle executes a less than $90^{\circ}$ roll and a negative or positive normal acceleration.

In order to execute these commands, the vehicle requires a control system to convert them into actuators commands. The other objective of the control system is the improvement of the dynamic stability of the vehicle. This is accomplished by augmenting the aerodynamic damping derivatives by rate feedback loops. The designed control system is as follow

$$
\begin{aligned}
& \delta a=-k_{a 1} p-k_{a 2}\left(\phi-\phi_{c}\right) \\
& \delta e=-k_{e 1} q-k_{e 2} a_{z}-k_{e 3} \int\left(a_{z_{c}}-a_{z}\right) d t \\
& \delta r=-k_{r 1} r-k_{r 2} a_{y}+k_{r 3} \int a_{y} d t \\
& \delta t=-k_{t 1}\left(a_{x_{c}}-a_{x}\right)-k_{t 2} \int\left(a_{x_{c}}-a_{x}\right) d t
\end{aligned}
$$

where $k_{a 1}, k_{a 2}, k_{e 1}, k_{e 2}, k_{e 3}, k_{r 1}, k_{r 2}, k_{r 3}, k_{t 1}, k_{t 2}$ are all the control system gains.

\section{Simulation \& RESUlts}

To verify the performance of the leader following controller, a six-degree-of-freedom simulation for a level turn scenario was developed by using $\mathrm{MATLAB}^{\circledR} /$ Simulink. The scenario constitutes of a micro air vehicle (follower) and a flying object (leader). The general specifications of the vehicle are as follow: $m=0.445 \mathrm{~kg}, \quad I_{x x}=0.002 \mathrm{~kg} \cdot \mathrm{m}^{2}, \quad I_{y y}=0.008 \mathrm{~kg} \cdot \mathrm{m}^{2}$, $I_{z z}=0.01 \mathrm{~kg} \cdot \mathrm{m}^{2}, \quad I_{x z}=2.10 e-4 \mathrm{~kg} \cdot \mathrm{m}^{2}, \quad b=0.61 \mathrm{~m}$, $S=0.089 \mathrm{~m}^{2}, \quad \bar{c}=0.147 \mathrm{~m}, \quad V_{\text {cruise }}=15 \mathrm{~m} / \mathrm{s}$, $T_{\max }=3.2 \mathrm{~N}$, the control surface deflections are $\pm 15^{\circ}$, and 

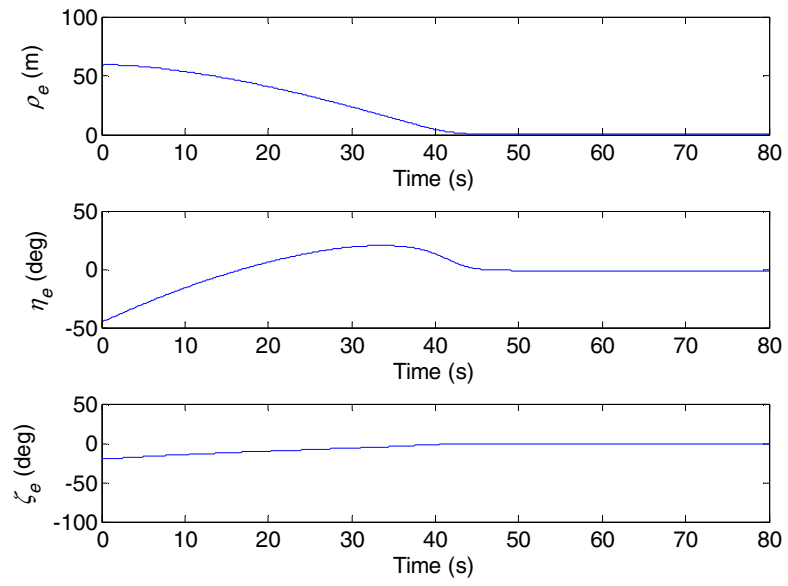

Fig. 3. Relative distance, azimuth angle, and elevation angle errors vs. time.
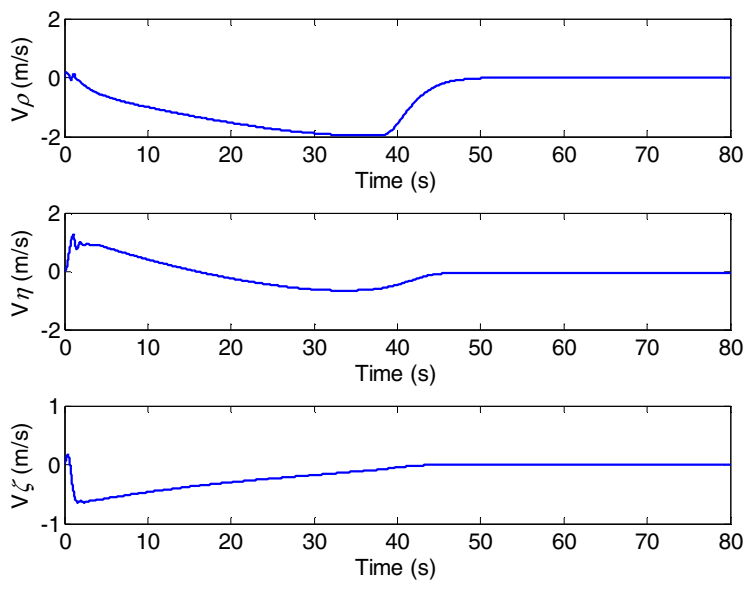

Fig. 4. Components of differential inertial velocity vs. time.

the control surface rate limits are considered $\pm 50 \%$. In the simulation, the parameters of the leader following controller are selected as $k_{1}=0.4, k_{2}=5, k_{3}=-1, \beta_{1}=0.6$, $\beta_{2}=5, \beta_{3}=5, \varepsilon_{1}=1, \varepsilon_{2}=2, \varepsilon_{3}=2, d=1 \mathrm{~m}$, and $\eta_{d}=0$, and $\zeta_{d}=0$.

The trajectories of the vehicle and leader are shown in Fig. 2. The leader moves in a circular path and the vehicle starts off the path but approaches the leader in the desired direction and follows the leader from the rear. The relative distance error between the vehicle and the leader, the azimuth angle, and the elevation angle are illustrated in Fig.
3 respectively. It can be seen that they are stabilized in the finite time. Fig. 4 illustrates the components of differential inertial velocity in the line of sight coordinate system. These rates are also stabilized by the controller in the finite time.

\section{CONCLUSION}

This paper presented the development of the leader following control used for the autonomous air robots. The state-space equations developed here can be used as a framework for the future studies on this topic. The sliding mode control was used to design the leader following controller. The equivalent control and saturation function were employed in order to eliminate the chattering phenomenon. The simulation results of the level turn scenario verified the controller performance in presence of leader's maneuvers.

\section{REFERENCES}

[1] F. Giulietti, L. Pollini, and M. Innocenti, "Autonomous formation flight," IEEE Contr. Syst. Mag., vol. 20, Dec. 2000

[2] J. Desai, J. P. Ostrowski, and V. Kumar, "Modeling and control of formations of nonholonomic mobile robots," IEEE Trans. Robot. Automat., vol. 17, June 2001.

[3] T. Soleymani and F. Saghafi, "Behavior-Based Acceleration Commanded Formation Flight Control," in Proc. IEEE ICCAS Conf., Gyeonggi-do, Dec. 2010.

[4] J. Nalepka and J. Hinchman, "Automated Aerial Refuling: Exteding the Effectiveness of UAVs," AIAA, Aug. 2005.

[5] I. Kaminer, E. Hallberg, and C. Silvestre, "Trajectory Tracking for Autonomous Vehicles: An Integrated Approach to Guidance and Control," AIAA Journal of Guidance, Control, and Dynamics, vol. 21, no. 1, Jan-Fab 1998.

[6] W. Ren and R. W. Beard, "Trajectory Tracking for Unmanned Air Vehicles with Velocity and Heading Rate Constraints," IEEE Trans. Contr. Syst. Tech., vol. 12, no.5, Sep 2004.

[7] M. Innocentri, L. Pollini, and D. Turra, "A Fuzzy Approach to the Guidance of Unmanned Air Vehicles Tracking Moving Targets," IEEE Trans. Contr. Syst. Tech., vol. 16, no. 6, Nov. 2008.

[8] S. Park, J. Deyst, and J. How, "A New Nonlinear Guidance Logic for Trajectory Tracking," AIAA 2004-4900, Aug. 2004.

[9] Y.GU, B. Seanor, G. Campa, M R. Napolitano, L. Rowe, S. Gururajan, and S. Wan, "Design and Flight Testing Evaluation of Formation Control Laws," IEEE Trans. Contr. Syst. Tech., vol. 14, no.6, Nov 2006

[10] H. Khalil, "Nonlinear Systems," Prentice Hall, New Jersey, Third Ed., 2002.

[11] D.Chwa, "Sliding-Mode Tracking Control of Nonholonomic Wheeled Mobile Robots in Polar Coordinates," IEEE Trans. Contr. Syst. Tech., vol. 12, no. 4, Jul. 2004.

[12] F. Morbidi and D. Prattichizzo. Sliding mode formation tracking control of a tractor and trailer-car system. In Proc. Robotics: Science and Systems III. MIT press, Cambridge, MA, 2007.

[13] P. Zipfel, "Modeling and Simulation of Aerospace Vehicle Dynamics," AIAA Education Series, Virginia, Second Ed., 2007. 